\title{
SUMMATION METHODS AND UNIQUENESS IN VILENKIN GROUPS
}

\author{
D. J. GRUBB
}

(Communicated by Richard R. Goldberg)

\begin{abstract}
It is shown that the $\sigma$-compact $U$-sets for two different summation methods on Vilenkin groups are the same.
\end{abstract}

Let $G$ be a Vilenkin group, i.e. a compact, totally disconnected, abelian, metric group, and let $\left\{H_{n}\right\}_{n=0}^{\infty}$ be a decreasing sequence of clopen subgroups of $G$ forming a neighborhood base at the identity of $G$. Let $\Gamma$ be the Pontryagin dual of $G$ and let $K_{n}=\operatorname{Ann}\left(\Gamma, H_{n}\right)$ be the annihilator of $H_{n}$ in $\Gamma$. Then $\left\{K_{n}\right\}_{n=0}^{\infty}$ is an increasing sequence of finite subgroups of $\Gamma$ whose union is all of $\Gamma$.

Let $A(G)$ be the algebra of absolutely convergent Fourier series, $P M(G)$ the space of pseudomeasures, and $P F(G)$ the space of pseudofunctions, on $G$. Then $A(G) \simeq l_{1}(\Gamma), P M(G) \simeq l_{\infty}(\Gamma)$, and $P F(G) \simeq c_{0}(\Gamma)$, where the isomorphisms are all written as $S \mapsto \hat{S}$.

If $S \in P M(G)$, we may define the $n$th partial sum of the Fourier series of type 1 of $S$ at a point $x$ in $G$ by

$$
s_{n}(S, x)=s_{n}(S)(x)=\left\langle S, \xi_{x H_{n}}\right\rangle / \lambda\left(H_{n}\right)=\sum_{\gamma \in K_{n}} \hat{S}(\gamma) \gamma(x),
$$

where $\langle S, f\rangle$ realizes the duality $P M(G) \simeq A(G)^{*}$ of Banach spaces. This summation method is investigated in [1 and 2].

In [3] Vilenkin showed how to enumerate $\Gamma$ as $\left\{\gamma_{n}\right\}_{n=0}^{\infty}$ in such a way that

(*) for fixed $m \geq 0$, the sequence $\left\{\gamma_{n}\right\}_{n=0}^{\infty}$ fills cosets of $K_{m}$ successively. Also $\gamma_{0}=1$.

Using this enumeration, we may construct another summation method for trigonometric series. Simply investigate the series

$$
\sum_{n=0}^{\infty} \hat{S}\left(\gamma_{n}\right) \gamma_{n}(x)
$$

for $S \in P M(G)$. We call partial sums of this series partial sums of type 2 , and this the Fourier series of $S$ of type 2.

We call a subset $E$ of $G$ a set of uniqueness or $U$-set of type 1 (resp. type 2) if the only Fourier series of type 1 (resp. type 2) of a pseudofunction converging to 0 everywhere except, possibly, on $E$ is the zero series. Notice that pseudofunctions are used in this definition and not pseudomeasures. This is important since otherwise there would be no $U$-sets of type 1 . (See [1] for a discussion.)

Received by the editors December 2, 1986.

1980 Mathematics Subject Classification (1985 Revision). Primary 43A55; Secondary 42C25.

Key words and phrases. Set of uniqueness, Vilenkin group. 
The object of this paper is a demonstration that for $\sigma$-compact subsets of $G$, the $U$-sets of type 1 are exactly the $U$-sets of type 2 . Because a partial sum of type 1 is a partial sum of type 2 (recall $(*)$ and $\gamma_{0}=1$ ), a $U$-set of type 1 is a $U$-set of type 2 , even if not $\sigma$-compact. It is also easy to show that the empty set is a $U$-set of type 1 , and it is known that countable unions of closed $U$-sets of type 1 are again $U$-sets of type 1. (See [1 and 2].) It is also known that closed $U$-sets of type 1 are exactly those closed sets which support no pseudofunction in the distributional sense [2]. Thus three different definitions of the concept of a $U$-set coincide for closed sets.

A key step in our program is the following proposition. For the case when $G=\Pi \mathbf{Z} /(2)$, see [4 and $\mathbf{5}]$.

THEOREM 1. Let $S \in P F(G)$ and let $x H_{m}$ be a basic open set in $G$. Then the type 1 Fourier series of $S$ converges to 0 on $x H_{m}$ if and only if the type 2 Fourier series of $S$ converges to 0 on $x H_{m}$.

PROOF. Since type 1 partial sums are type 2 partial sums, one direction is trivial.

Now assume that $s_{n}(S, y)$ converges to 0 for every $y$ in $x H_{m}$. Let $Q$ be a representative set in $\Gamma$ for the cosets of $K_{m}$, where $K_{m}$ is the annihilator of $H_{m}$. Then for $y$ in $H_{m}$ and $n \geq m$, write

$$
\begin{aligned}
s_{n}(S, x y) & =\sum_{\gamma \in K_{n}} \hat{S}(\gamma) \gamma(x y)=\sum_{\phi \in Q \cap K_{n}} \sum_{\eta \in K_{m}} \hat{S}(\phi \eta)(\phi \eta)(x y) \\
& =\sum_{\phi \in Q \cap K_{n}}\left[\sum_{\eta \in K_{m}} \hat{S}(\phi \eta) \eta(x)\right] \phi(y) .
\end{aligned}
$$

Since the Pontryagin dual of $H_{m}$ is isomorphic to $\Gamma / K_{m}$, this last sum may be regarded as a partial sum of a type 1 Fourier series on $H_{m}$. Since $s_{n}(S, x y)$ converges to 0 for all $y \in H_{m}$, and since the empty set is a $U$-set of type 1 in $H_{m}$, we get

$$
\sum_{\eta \in K_{m}} \hat{S}(\phi \eta) \eta(x)=0 \quad \text { for } \phi \in Q .
$$

If $y \in x H_{m}, y H_{m}=x H_{m}$, so the above holds with $y$ in place of $x$. Rewriting gives

$$
\sum_{\gamma \in \phi K_{m}} \hat{S}(\gamma) \gamma(y)=0 \quad \text { for } y \in x H_{m} \text { and } \phi \in Q .
$$

Now, if $n \geq 0$ is given, then $\left\{\gamma_{0}, \ldots, \gamma_{n}\right\}$ is a union of cosets of $K_{m}$ except for a "tail" $\left\{\gamma_{p}, \ldots, \gamma_{m}\right\}$ lying entirely in one coset of $K_{m}$, since the sequence $\left\{\gamma_{j}\right\}_{1}^{\infty}$ fills up cosets of $K_{m}$ successively. This fact, with (1) shows

$$
\begin{aligned}
\left|\sum_{k=0}^{n} \hat{S}\left(\gamma_{k}\right) \gamma_{k}(y)\right| & =\left|\sum_{k=p}^{n} \hat{S}\left(\gamma_{k}\right) \gamma_{k}(y)\right| \leq(n-p+1) \sup |\hat{S}(\gamma)| \\
& \leq \operatorname{card}\left(K_{m}\right) \sup |\hat{S}(\gamma)| \rightarrow 0 \quad \text { for } y \in x H_{m},
\end{aligned}
$$

where the supremum is over a coset of $K_{m}$ which goes to infinity in $\Gamma$ as $n$ goes to infinity. This completes the proof of the proposition. 
COROLlaRY 2. A $\sigma$-compact subset of $G$ is a $U$-set of type 1 if and only if it is a $U$-set of type 2 .

ProOF. As noted above, any $U$-set of type 1 is a $U$-set of type 2 automatically. The theorem shows that a closed $U$-set of type 2 is a $U$-set of type 1 . Now, if $E=\bigcup_{n=0}^{\infty} E_{n}$ is a $\sigma$-compact $U$-set of type 2 , with each $E_{n}$ closed in $G$, then each $E_{n}$ is a $U$-set of type 2 and thus of type 1 . Since countable unions of $U$-sets of type 1 are $U$-sets of type 1 (see [1 or 2]), $E$ is a $U$-set of type 1.

\section{BIBLIOGRAPHY}

1. D. J. Grubb, Sets of uniqueness in compact, 0-dimensional, metric groups, Trans. Amer. Math. Soc. 301 (1987), 239-249.

2. _ Completeness and uniqueness on compact, 0-dimensional, metric spaces, unpublished.

3. N. Ja. Vilenkin, On a class of complete orthonormal systems, Izv. Akad. Nauk SSSR 11 (1947), 363-400; English transl., Amer. Math. Soc. Transl. 28 (1963), 1-35.

4. K. Yoneda, Sets of multiplicity on the dyadic group, Acta Math. Hungar. 41 (1983), 195-200.

5. V. A. Skvorcov, Example of a Walsh series with a subsequence of partial sums converging everywhere to 0, Mat. Sb. 97 (1975), 517-539.

Department of Mathematical Sciences, Northern Illinois University, DEKALB, ILLINOIS 60115 